\title{
An Evaluation of the Adverse Events Following Voluntary Medical Male Circumcision Surveillance System in Zvimba District, Zimbabwe, 2020
}

\author{
Tendai Hlabangana1, Owen Mugurungi², Emmanuel Govha' ${ }^{1}$, Tsitsi P. Juru' ${ }^{1}$, Notion T. Gombe1,3, \\ Addmore Chadambuka ${ }^{1 *}$, Maurice Omondi ${ }^{4}$, Mufuta Tshimanga ${ }^{1}$ \\ ${ }^{1}$ Department of Family Medicine, Global and Public Health Unit, University of Zimbabwe, Harare, Zimbabwe \\ ${ }^{2}$ AIDS and TB Programmes Directorate, Harare, Zimbabwe \\ ${ }^{3}$ African Field Epidemiology Network, Harare, Zimbabwe \\ ${ }^{4}$ African Field Epidemiology Network, Nairobi, Kenya \\ Email: tendaihlabangana@gmail.com, mugurungi@gmail.com, govha86@yahoo.com, tsitsijuru@gmail.com, \\ ntgombe@gmail.com, ^achadambuka1@yahoo.co.uk, mowiny@feltp.org.ke, tshimangamufuta@gmail.com
}

How to cite this paper: Hlabangana, T., Mugurungi, O., Govha, E., Juru, T.P., Gombe, N.T., Chadambuka, A., Omondi, M. and Tshimanga, M. (2022) An Evaluation of the Adverse Events Following Voluntary Medical Male Circumcision Surveillance System in Zvimba District, Zimbabwe, 2020. World Journal of AIDS, 12, 1-19.

https://doi.org/10.4236/wja.2022.121001

Received: January 13, 2022

Accepted: February 27, 2022

Published: March 2, 2022

Copyright $\odot 2022$ by author(s) and Scientific Research Publishing Inc. This work is licensed under the Creative Commons Attribution International License (CC BY 4.0).

http://creativecommons.org/licenses/by/4.0/ (c) (i) Open Access

\begin{abstract}
Background: The Adverse Events following Voluntary Medical Male Circumcision Surveillance System (VMMC AESS) serves to identify adverse events (AEs) for safe provision of VMMC. In 2019, Zvimba District reported two adverse events out of 542 circumcisions translating to a $0.4 \% \mathrm{AE}$ rate against an acceptable threshold of $2 \%$ and this is suggestive of underreporting. We evaluated the system to verify existence of underreporting of AEs, assess the surveillance system attributes and evaluate data quality. Methods: A descriptive cross-sectional study was conducted using the updated Center for Disease Control (CDC) guidelines for surveillance system evaluation. We proportionately sampled VMMC client intake records and purposively selected health workers involved in the VMMC programme into the study. An interviewer-administered questionnaire was used to collect data from 14 - 30 December 2020 on knowledge of the VMMC AESS among health care workers and surveillance system attributes. We used a checklist to check for data quality. Data were analysed using Epi Info 7 statistical software. Results: Of the 31 health workers that participated in the study, $21(68 \%)$ had fair knowledge of the surveillance system. Out of 384 records reviewed, 104 had missing data on AE monitoring days 7 and 42. Sixteen (52\%) of participants regarded filling $\mathrm{AE}$ reporting forms as difficult and the process as time-consuming. The surveillance system was not representative as only two out of eight facilities were conducting outreaches in hard-to-reach areas. Conclusion: There was possi-
\end{abstract}


ble underreporting of adverse events as about a quarter of reviewed records had missing data on AE monitoring days and the system's performance was threatened by poor data quality and unavailability of reporting forms. The system was rendered acceptable though unstable, not representative and not simple and the health workers were not making use of the surveillance system. We recommended retraining on the surveillance system for all health care workers involved in VMMC in Zvimba District.

\section{Keywords}

Surveillance, Adverse Events, System, Circumcision, Evaluation

\section{Introduction}

Voluntary Medical Male Circumcision (VMMC) is defined as the voluntary removal of the prepuce (foreskin) of the penis for medical reasons [1]. Male circumcision (MC) reduces the risk of female-to-male HIV transmission by up to $60 \%$ and since 2007, the World Health Organization (WHO) and the US President's Emergency Plan for AIDS Relief (PEPFAR) began supporting the implementation of voluntary medical male circumcision(VMMC) programmes across 14 priority African countries including Zimbabwe [2] [3].

Zimbabwe embraced voluntary medical male circumcision in 2009 as part of a comprehensive package of HIV prevention and treatment services [4]. The adverse events following voluntary medical male circumcision surveillance system in Zimbabwe were adopted at the inception of the programme in the country in 2009 to ensure safe and quality VMMC service provision. It is a paper-based recording and reporting system and the flow of information in the surveillance system is shown (Figure 1). The flow of information in the surveillance system as depicted in Figure 1, begins with case-based identification of adverse events at the health facility where four copies of the VMMC adverse event reporting form are completed by the health care workers involved in VMMC (for moderate and severe AEs). One copy of the form is kept at the health facility at which the $\mathrm{AE}$ is identified with the rest of the forms being forwarded to the district office, the provincial medical directorate's office and subsequently to the head office. Information about reportable AEs from the health care facilities is reported to the district office within 24 hours and a summary of monthly AEs is reported to the technical partner within 30 days. Severe A/Es are supposed to be reported to the national office within 48 hours. Whilst there are studies that have been done before in Zimbabwe in evaluating the adverse events in VMMC surveillance system, these studies have been specific to the PrePex device [2] [3]. There has been a gap in evaluating the effectiveness of the surveillance system in the VMMC programme as a whole when other circumcision methods which are currently in use in the country such as the dorsal slit and surgical resection methods are utilised [5]. 
MoHCC Head Office - National VMMC Coordinator

Consolidation of information on Adverse Events following VMMC reports from all provinces. *(Severe Adverse Events following VMMC to reach National Office within 48 hours)

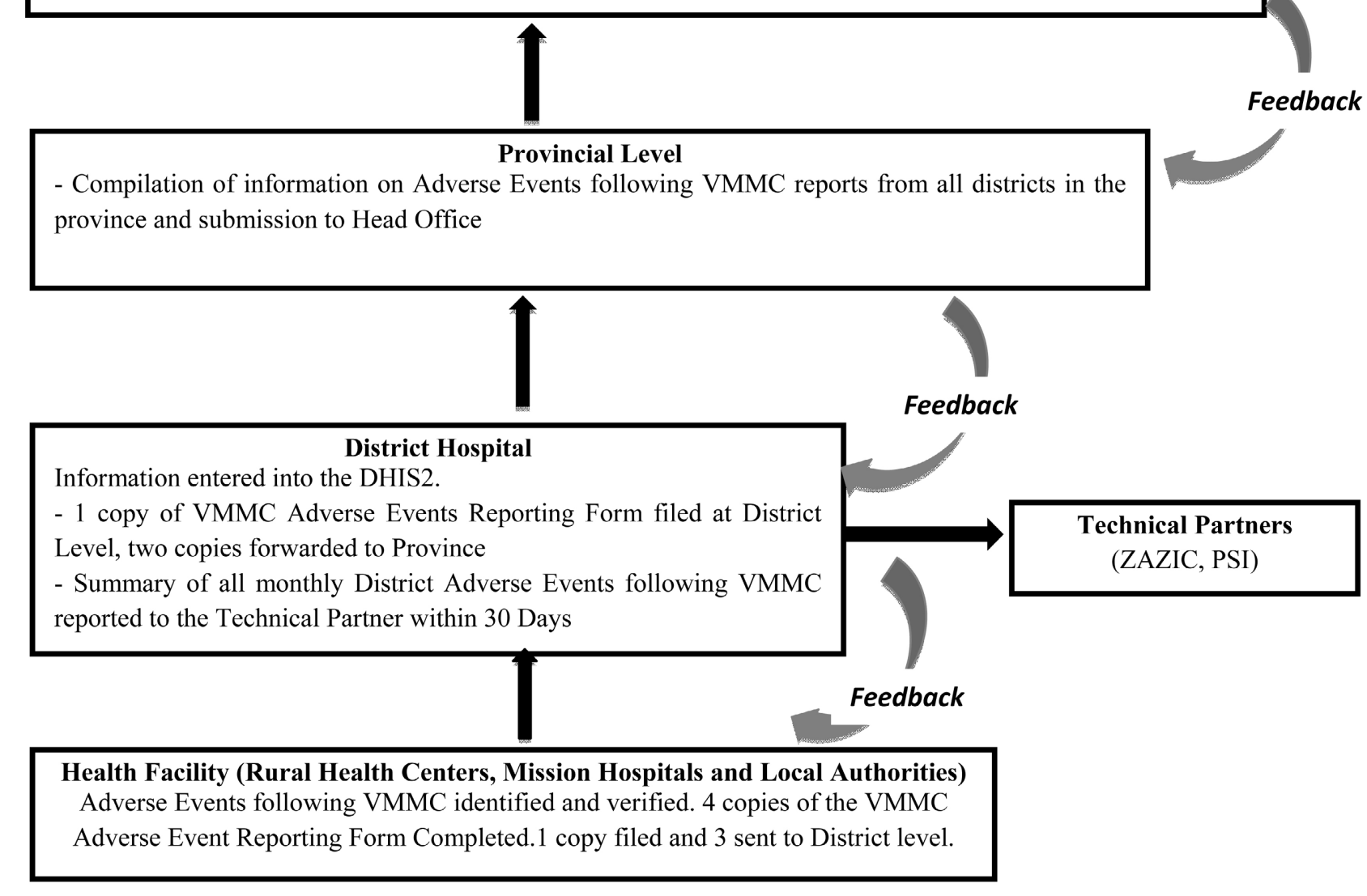

Figure 1. Flow of information in the adverse events following voluntary medical male circumcision surveillance system in Zimbabwe (Source: National VMMC Programme, Harare, Zimbabwe).

Zvimba District had one of the lowest notifications of adverse events following voluntary medical male circumcision in Mashonaland West Province in 2019. There were only two adverse events reported from 542 circumcisions conducted, accounting for $0.4 \%$ against an acceptable threshold of $2 \%$ for adverse event rates. These statistics are suggestive of underreporting of adverse events in the district. We evaluated the surveillance system to verify the existence of underreporting of adverse events and to ensure that the surveillance system attributes are functioning effectively so that no adverse events are missed should they occur.

\section{Methods}

\section{Study Design}

We conducted a descriptive cross-sectional study in Zvimba District from using the updated Centre for Disease Control (CDC) guidelines for surveillance system evaluation and collected data from 14-30 December 2020 [6].

\section{Study Setting}


We conducted the study at all the eight government-owned health care facilities currently offering VMMC in Zvimba District in Mashonaland West Province, Zimbabwe. The health facilities are Banket District Hospital, Father O'Hea Mission Hospital, Raffingora Rural Hospital, Darwendale Rural Hospital, Zvimba Rural Hospital, Mt Hampden Clinic, Madzorera Clinic and Tobacco Research Board (TRB) Clinic. According to the District Population Projections report of 2020, the current population of Zvimba District is 317,591, with 161,660 (50.9\%) males and 155,931 (49.1\%) females [7]. According to the Sustainability Transition Implementation Plan for Voluntary Medical Male Circumcision report for 2019-2021, the VMMC programme targets males between the ages of $10-29$ years [5]. HIV and AIDS is major public health threat in the district with 2018 estimates showing that there was approximately 41,850 people living with HIV in the district and approximately 831 new HIV infections and 710 HIV-related deaths recorded in the district in the same year [8].

\section{Sample Size and Assumptions}

We calculated a minimum sample size of 44 health care workers utilizing Dobson's formula

$$
n=Z_{a}^{2} p(1-p) / d^{2}
$$

where $Z_{a}=1.96, d=0.05$ and $p=0.97$ based on assumptions from an unpublished study by Dube et al. (2017) in Chegutu District, Zimbabwe where $97 \%$ of the health workers in the study had fair knowledge about the adverse events following voluntary medical male circumcision surveillance system at $95 \%$ confidence interval, $5 \%$ margin of error and factoring in an attrition rate of $10 \%$ [9].

The minimum sample size of 250 records for review was calculated based on assumptions that completeness of filling forms was $80 \%$ (expected national target for completeness), precision of the study at $20 \%$, Confidence level $95 \%$ based on a study by Dube et al. (2017) in Chegutu District, Zimbabwe [9].

\section{Sampling and Study Participants}

The primary respondents were health care workers directly involved in voluntary medical male circumcision namely registered general nurses (RGNs), primary care nurses (PCNs) and government medical officers (GMOs). We recruited all the eight health facilities currently offering VMMC in the district into the study. We purposively selected health care workers directly involved in the circumcision programme who were on duty during data collection and proportionate random sampling of VMMC client intake records were done at each health facility.

\section{Data Collection and Analysis}

A pre-tested interviewer-administered questionnaire (see Appendix 1) was used to collect data from health workers on VMMC surveillance system attributes namely stability, simplicity, flexibility, acceptability and representativeness. We also collected data on knowledge on the surveillance system from health care workers. The questionnaire was pretested and adjusted by interviewing five health care workers involved in VMMC (10\% of the sample size) at Norton Hospital 
which is about $39 \mathrm{~km}$ from the capital city Harare. We chose Norton Hospital as it has a similar setup with the health care facilities offering VMMC in Zvimba District. Adjustments that were made included adding a section that assessed health care workers' knowledge on classification of adverse events according to type, severity and timing. We reviewed VMMC client intake forms covering the period from 1 January 2019 to 31 December 2020 at each health facility to check for data quality and to check if there were any unreported adverse events.

\section{Measurement of Variables}

\section{Knowledge of health care workers}

We assessed health workers' awareness and understanding of the components of the AEs following VMMC surveillance system by asking participants to answer questions regarding VMMC AEs surveillance. The questions centred on ability to state the objectives for AEs surveillance, classification of the AEs, the reporting channel, the time frame to report and the criteria for coding of adverse events. A three-point Likert scale was used to grade knowledge level among health care workers.

\section{Usefulness}

This is a measure of whether the system contributes to the prevention and control of adverse health-related events, including an improved understanding of the public health implications of such events [6]. Usefulness was assessed by ascertaining whether there were any meetings done to review AE data and whether there were any public health actions that were implemented based on the surveillance system findings.

\section{Data Quality}

Data quality refers to the completeness and accuracy of the data recorded in the surveillance system. We measured data quality by checking the completion of data on various fields on the form and the capturing of data on monitoring AEs on days 2, 7 and 42 of the review periods.

\section{Simplicity}

This attribute refers to the structure and the ease of operation of the surveillance system [6]. We assessed simplicity by looking at the proportions of health workers who had ever identified an adverse event and completed adverse event reporting forms. We also evaluated simplicity by assessing whether the health care workers felt they needed further training on filling $\mathrm{AE}$ data and how they regarded the simplicity of filling out AE reporting forms.

\section{Acceptability}

Acceptability refers to the willingness of persons and organizations to participate in the surveillance system [6]. We assessed acceptability by ascertaining willingness to continue participating in the surveillance system by health workers among other indicators. We assessed acceptability objectively by checking for the availability of VMMC monthly return forms that were submitted to the next level.

\section{Stability}


Stability refers to the reliability (i.e. the ability to collect, manage, and provide data properly without failure) and availability of resources needed for the surveillance process [6]. We assessed stability by looking at the availability of resources required to run the surveillance system and the adequacy of knowledge of health care workers on adverse events data reporting. Objectively, we used a checklist to assess the availability of resources such as functional vehicles, stationery and communication tools at the facilities.

\section{Representativeness}

This measures whether the surveillance accurately describes the occurrence of a health-related event over time and its distribution in the population by place and person [6]. We assessed representativeness by looking at the availability of VMMC outreaches in hard-to-reach areas and whether there were any private health facilities offering VMMC.

\section{Flexibility}

A flexible public health surveillance system can adapt to changing information needs or operating conditions with little additional time, personnel, or allocated funds. The flexibility of the VMMC AE surveillance system was assessed by evaluating whether the VMMC personnel would want to in-cooperate any other additional AEs and if there were any current adjustments that had been done to accommodate any other A/Es.

\section{Data Analysis}

Epi Info 7.2.4.0 ${ }^{\mathrm{TM}}$ (CDC, 2020) statistical package was used to capture and analyze the data from questionnaires. Means and frequencies were calculated using the same software. A three-point Likert scale was used to rate health care workers' knowledge of the surveillance system. Those who answered two or fewer questions correctly out of six questions were classified as having low or poor knowledge level, three to four out of six questions as having fair knowledge level and those who answered five to six questions correctly and were classified as having good knowledge level of the surveillance system.

\section{Ethical Considerations}

We obtained permission to conduct the study from the Health Studies Office, the Director for AIDS \& TB Unit, Provincial Medical Directorate (PMD) for Mashonaland West Province and District Medical Officer (DMO) for Zvimba District. Informed written consent was obtained from all the interviewees and confidentiality was ensured. Since data collection took place during the COVID-19 pandemic, the essential infection prevention protocols were strictly adhered to.

\section{Results}

Demographic characteristics of VMMC health care workers in Zvimba District, 2020

Thirty-one health care workers were recruited into the study with females constituting $16(52 \%)$ and males 15 (48\%) of the study participants. The majority $18(58 \%)$ of study participants were Registered General Nurses (RGNs) and 
the Government Medical Officers (GMO) constituted the least, with 3 (10\%) participants. The median age of the study participants was 38 years $\left(\mathrm{Q}_{1}=35, \mathrm{Q}_{3}\right.$ $=42)$. The median time in service for participants was 12 years $\left(Q_{1}=10, Q_{3}=\right.$ $15)$ and the median time in-service involved with $\mathrm{VMMC}$ was 4 years $\left(\mathrm{Q}_{1}=2, \mathrm{Q}_{3}\right.$ =5) (Table 1).

Knowledge of VMMC health care workers on the surveillance system

All the participants were aware of the VMMC AEs surveillance system and could state at least one objective of the surveillance system. However, on classification of severity and coding of AEs, less than half of participants 13 (42\%) gave the correct description on both criteria. Overall, using a three-point Likert scale, the majority 21 (68\%) of the health care workers had fair knowledge whilst $6(19 \%)$ had good knowledge and only $4(13 \%)$ had poor knowledge of the surveillance system (Table 2).

Stability of the surveillance system in Zvimba District, 2020

More than two-thirds 21 (68\%) of the participants felt that they still needed further training with special mention on the identification and documentation of AEs. Less than a quarter $6(19 \%)$ of the participants believed that the resources

Table 1. Demographic characteristics of voluntary medical male circumcision health care workers in Zvimba District, Zimbabwe, 2020.

\begin{tabular}{|c|c|c|}
\hline Aspect & $\begin{array}{c}\text { Frequency } \\
\mathrm{n}=31\end{array}$ & Percentage \\
\hline \multicolumn{3}{|l|}{ Gender } \\
\hline Male & 16 & 51.6 \\
\hline Female & 15 & 48.4 \\
\hline \multicolumn{3}{|l|}{ Designation } \\
\hline Registered General Nurse (R.G.N) & 18 & 58.1 \\
\hline Primary Care Nurse (P.C.N) & 10 & 32.3 \\
\hline Government Medical Officer (G.M.O) & 3 & 9.7 \\
\hline \multicolumn{3}{|l|}{ Age groups } \\
\hline $25-29$ years & 3 & 9.7 \\
\hline 30 - 34 years & 5 & 16.1 \\
\hline 35 - 39 years & 13 & 41.9 \\
\hline 40 - 44 years & 4 & 12.9 \\
\hline 45 - 49 years & 5 & 16.1 \\
\hline $50+$ years & 1 & 0.03 \\
\hline Median age & $38\left(\mathrm{Q}_{1}=35, \mathrm{Q}_{3}=42\right)$ & \\
\hline Median age in service & $12\left(\mathrm{Q}_{1}=10, \mathrm{Q}_{3}=15\right)$ & \\
\hline Median age involved in VMMC & $4\left(Q_{1}=2, Q_{3}=5\right)$ & \\
\hline
\end{tabular}


Table 2. Knowledge of health care workers on the adverse events following voluntary medical male circumcision surveillance system in Zvimba District, Zimbabwe, 2020.

\begin{tabular}{ccc}
\hline Knowledge Attribute & $\begin{array}{c}\text { Frequency } \\
(\mathrm{n}=31)\end{array}$ & Percentage \\
\hline $\begin{array}{c}\text { Aware of the AEs following VMMC surveillance system } \\
\text { Knows objective of AEs following VMMC surveillance } \\
\text { system }\end{array}$ & 31 & 100 \\
$\begin{array}{c}\text { Knows timeline for reporting AEs to next level } \\
\begin{array}{c}\text { Correctly describe criteria for classifying AEs } \\
\text { (based on type, severity, and timing) } \\
\text { Correctly describe AE coding A-C } \\
\text { (timing of when AE occurs) }\end{array}\end{array}$ & 29 & 100 \\
$\begin{array}{c}\text { Correctly describe severity criteria of AEs } \\
\text { (i.e., mild, moderate, severe) }\end{array}$ & 15 & 48.4 \\
3-Point Likert Scale & 13 & 41.9 \\
Poor & 13 & 41.9 \\
Fair & 4 & 12.9 \\
Good & 6 & 67.7 \\
\hline
\end{tabular}

for running the surveillance system were adequate (Table 3). All 8 facilities had functional phones and VMMC registers available on site. The national adverse events classification guidelines were found displayed at $5 / 8$ of the health facilities whilst adverse event reporting forms were available at only $3 / 8$ of the health facilities. Functional vehicles were available at $4 / 8$ of the health facilities and those vehicles were not solely for VMMC activities as they are assigned to other activities as well.

\section{Acceptability of the surveillance system in Zvimba District, 2020}

All the participants $31(100 \%)$ reported that they were the ones responsible for compiling $\mathrm{AE}$ data and expressed willingness to continue participating in the surveillance system and forwarding reports on VMMC adverse events monitoring to the next level. Acceptability of the surveillance system was also confirmed by the fact that all health facilities were compiling VMMC monthly return forms which were being submitted timeously to the next reporting level.

\section{Simplicity of the surveillance system in Zvimba District, 2020}

Only 10 (32\%) of the participants reported that they had ever identified an adverse event following VMMC whilst 27 (87\%) of the participants reported that they had never completed adverse events form. More than half of the participants $22(71 \%)$ highlighted that it may take an average of $11-20$ minutes to complete the A/E reporting form and 16 (52\%) of the participants regarded filling $\mathrm{AE}$ reporting form as difficult. The findings on the assessment of simplicity attribute of the surveillance system are highlighted in (Table 4).

\section{Flexibility}


Table 3. Stability of the adverse events following voluntary medical male circumcision surveillance system in Zvimba District, Zimbabwe, 2020.

\begin{tabular}{|c|c|c|}
\hline Aspect & $\begin{array}{l}\text { Frequency } \\
\qquad \mathrm{n}=31\end{array}$ & Percentage \\
\hline Ever trained in VMMC & 31 & 100 \\
\hline \multicolumn{3}{|l|}{ Last time trained } \\
\hline Last 12 months & 10 & 32.3 \\
\hline 13 - 24 months & 14 & 45.2 \\
\hline $25-36$ months & 4 & 12.9 \\
\hline$>36$ months & 3 & 9.7 \\
\hline \multicolumn{3}{|l|}{ Type of training } \\
\hline Formal & 20 & 64.5 \\
\hline On the job & 11 & 35.5 \\
\hline Believes that training was adequate & 15 & 48.4 \\
\hline Still needs further training & 21 & 67.7 \\
\hline $\begin{array}{l}\text { Believes that resources for the surveillance } \\
\text { system are adequate }\end{array}$ & 6 & 19.4 \\
\hline \multicolumn{3}{|c|}{ Stability of Surveillance System by Resource Availability } \\
\hline Tool & $\begin{array}{l}\text { Health facilities } \\
\qquad \mathrm{n}=\mathbf{8}\end{array}$ & Percentage \\
\hline Functional phone & 8 & 100 \\
\hline VMMC registers & 8 & 100 \\
\hline AE classification guidelines & 5 & 62.5 \\
\hline $\mathrm{AE}$ reporting forms & 3 & 37 \\
\hline Functional vehicle & 4 & 50 \\
\hline
\end{tabular}

Table 4. Simplicity of the adverse events following voluntary medical male circumcision surveillance system in Zvimba District, Zimbabwe, 2020.

\begin{tabular}{ccc}
\hline Simplicity Attribute & $\begin{array}{c}\text { Frequency } \\
(\mathrm{n}=31)\end{array}$ & $\begin{array}{c}\text { Percentage } \\
\%\end{array}$ \\
\hline Ever identified an AE & 10 & 32.3 \\
Ever completed an AE form & 4 & 12.9 \\
Ever reported an AE case & 8 & 25.8 \\
Additional training required to \\
complete AE data & 23 & 74.2 \\
Time taken to complete AE Reporting form & & \\
5 - 10 mins & 8 & 25.8 \\
$11-20$ mins & 22 & 70.9 \\
\hline
\end{tabular}




\begin{tabular}{ccc}
\hline $\begin{array}{c}\text { More than } 20 \text { mins } \\
\text { Regard of difficulty of completing } \\
\text { AE reporting form } \\
\text { Very simple }\end{array}$ & 1 & 3.2 \\
Simple & 4 & \\
Difficult & 9 & 12.9 \\
Very difficult & 16 & 29.0 \\
\hline
\end{tabular}

There are no adjustments that have been made to accommodate any other adverse events at all health care facilities offering VMMC. A total of 30 (97\%) participants also highlighted that there were no other additional AEs that they would want to be monitored at their sites whilst one participant highlighted that they would want "psychological effects" monitored as an adverse event following VMMC.

\section{Data quality}

Client Intake forms at each health facility were completed by the nurse circumciser who is a registered general nurse (RGN). Out of the 384 client intake records reviewed, a total of 104 (27\%) had incomplete data on different sections. Of the 104 records with missing data 99 (95\%) had missing AE data on review day 42 and a small proportion 5 (5\%) had missing AE data on both days 7 and 42.

\section{Representativeness}

Out of the 11 private health facilities in Zvimba District, none were offering VMMC. All 8 public health care facilities involved in VMMC have hard-to-reach areas within their catchment areas, however only two were conducting mobile outreaches. Participants from these two public health care facilities reported that surveillance data from these outreach points was being reported but on physically checking there were no surveillance forms that were availed from those sites.

\section{Reasons for possible underreporting}

On perceived reasons for possible underreporting of $\mathrm{AE}$ data, more than three quarters 25 (81\%) of the study participants highlighted clients not attending reviews and $22(71 \%)$ highlighted lack of knowledge of AEs among health care workers. The least reported reason was a complex surveillance system as reported by $9(29 \%)$ of the study participants.

\section{Discussion}

Overall, knowledge of study participants on the adverse events following VMMC surveillance system was graded as fair on the Likert Scale. The majority of the participants were knowledgeable on examples of adverse events and timelines for reporting moderate or severe adverse events but scored poorly on describing 
the criteria used on the classification of adverse events. On the other hand, the surveillance system in Zvimba District was acceptable as all the participants in the study acknowledged that they are the ones responsible for filling AE data, was willing to continue participating in the surveillance system and timeously submitted surveillance data to the next level. The surveillance system was not simple as the majority of the study participants reported that they required additional training in documenting $\mathrm{AE}$ data and that they found the documentation process time-consuming. There were gaps in the adequate documentation of the adverse event monitoring as evidenced by some records not having data filled on all the days for the follow-up period.

Lack of knowledge by study participants in describing criteria for severity and coding of adverse events could be attributed to the unavailability of displayed adverse event classification guidelines at some of the health care facilities and the perceived inadequate training on the surveillance system as highlighted by the majority of the study participants. In a similar study by Dube et al. (2017) possible reasons that were given by the study participants as affecting the simplicity of the surveillance system was the lack of periodic refresher courses on identifying and documenting adverse events and the general lack of knowledge on VMMC adverse events by health care workers [9]. In Zvimba District, refresher VMMC training workshops course for health care workers were now limited due to decreasing funding for the programme as the country is now moving into the sustainability phase for the VMMC programme that is characterized by less reliance on donor funding. Surveillance systems should be as simple as possible whilst still meeting their objectives as a simple system is more likely to provide timely data and is generally more acceptable to staff members thus improving participation as highlighted by Matambo et al. (2016) [10]. The lack of resources due to decreasing funding for the VMMC programme could have also affected the capacity for health facilities to conduct outreaches in hard-to-reach areas and thus affecting the coverage of the VMMC programme as a whole.

Findings from this study also showed that the surveillance system was also not flexible as no other changes have been made to accommodate any other adverse events. This is similar to findings by Dube et al. (2017) who found out that there were no adjustments that had been done in accommodating other adverse events in the surveillance system [9]. Flexibility is necessary when changes occur in case definitions, reporting forms, adding new health events and procedures. This will ensure that the surveillance system remains relevant in the face of an ever-evolving healthcare delivery system.

One of the objectives of this study was to ascertain the reasons that were contributing to possible underreporting of AE data as highlighted by study participants. Findings from the key informants' interview acknowledged that there could be possible under-reporting of adverse events following VMMC in Zvimba due to various weaknesses in the surveillance system. Similar findings were highlighted by Marongwe et al. (2019) who reported that in Zimbabwe, previous studies on data quality and AE identification within the male circumcision pro- 
gram revealed challenges on correct and complete data and that the low $\mathrm{AE}$ rate in Zimbabwe may indicate potential AE underreporting [11]. Gadzayi et al. (2020) reported that some of the reasons for underreporting in a study of adverse events following immunization in a study that was conducted in Manicaland province included fear by healthcare workers of being blamed for causing the adverse event, clients poor knowledge of adverse events which results in them not reporting at facilities when an $\mathrm{A} / \mathrm{E}$ occurs and also the misclassification of events as mild [12]. Dube et al. (2017) state that reasons for under-reporting which are common include a surveillance system that does not capture marginalized high-risk groups, unawareness of the adverse events among health care workers and the clients not seeking medical care for various reasons. Clients do not seek medical care such as when they cannot take time off work, cannot access the hospital, or feel their illness does not warrant a trip to the hospital.

\section{Limitations}

We could not reach the minimum calculated sample size of 44 health care workers and this could have affected the power of the study.

\section{Conclusions}

From the records, we reviewed about a quarter of those records had incomplete data, especially for day 42 on the post-surgery monitoring of AEs. Health workers' knowledge was found to be fair on the three-point Likert scale but the majority scored poorly on describing the criteria used on classifying adverse events. All these factors could have contributed to underreporting of adverse events and thus affected the accuracy of the rates being reported. The A/Es following VMMC surveillance system in Zvimba District was found to be acceptable, but unstable, not flexible and not representative.

We recommended retraining of all health care workers involved in VMMC on the adverse events following VMMC surveillance system to capacitate health care workers to be competent in identifying and documenting adverse events that may occur during the VMMC programme.

\section{Acknowledgements}

We would like to acknowledge the AIDS and TB Programmes Directorate for the support during the study. To the Zimbabwe Field Epidemiology Training Program, thank you for financial and technical assistance.

\section{Conflicts of Interest}

The authors declare no competing interests whether financial or non-financial.

\section{Author Contributions}

TH: conception, design, acquisition, analysis and interpretation of data and drafting the manuscript. OM: conception, design, acquisition, analysis and interpretation of data and drafting the manuscript. NTG: conception, design, acquisi- 
tion, analysis, interpretation and reviewing manuscript draft for important intellectual content. EG: conception, design, acquisition, analysis and interpretation of data and reviewing manuscript draft for important intellectual content. AC: conception, design, acquisition, analysis, and interpretation of data and reviewing manuscript draft for important intellectual content. TJ: conception, design, acquisition, analysis, and interpretation of data and reviewing manuscript draft for important intellectual content. MO: reviewing manuscript draft for important intellectual content. MT: conception, design, acquisition, analysis and interpretation of data and reviewing manuscript draft for important intellectual content. All the authors read and approved the final manuscript.

\section{Funding}

This study was funded by the AIDS and TB Program and Health Studies Office.

\section{References}

[1] Dickson, K.E., Tran, N.T., Samuelson, J.L., Njeuhmeli, E., Cherutich, P., Dick, B., et al. (2011) Voluntary Medical Male Circumcision: A Framework Analysis of Policy and Program Implementation in Eastern and Southern Africa. PLOS Medicine, 8, e1001133. https://doi.org/10.1371/journal.pmed.1001133

[2] Adamson, P.C., Tafuma, T.A., Davis, S.M., Xaba, S. and Herman-Roloff, A. (2017) A Systems-Based Assessment of the PrePex Device Adverse Events Active Surveillance System in Zimbabwe. PLoS ONE, 12, e0190055. https://doi.org/10.1371/journal.pone.0190055

[3] Bochner, A.F., Feldacker, C., Makunike, B., Holec, M., Murenje, V., Stepaniak, A., et al. (2017) Adverse Event Profile of a Mature Voluntary Medical Male Circumcision Programme Performing PrePex and Surgical Procedures in Zimbabwe. Journal of the International AIDS Society, 20, Article No. 21394. https://doi.org/10.7448/IAS.20.1.21394

[4] Mapingure, M.P., Tapera, O., Munjoma, M., Mutedzi, B., Dhodho, E., Hatzold, K., et al. (2016) "Reaching the Higher Hanging Fruits": Tracking VMMC Uptake and Behavioural Determinants: Results from Multi-Year National Cross-Sectional Surveys in Zimbabwe. http://www.psi.org/publication

[5] Ministry of Health and Child Care (2018) VMMC Sustainable Transition Implementation Plan (2019-2021), Harare, Zimbabwe.

[6] American Psychological Association (2001) Updated Guidelines for Evaluating Public Health Surveillance Systems: Recommendations from the Guidelines Working Group. http://doi.apa.org/get-pe-doi.cfm?doi=10.1037/e548222006-001

[7] Chikeya, L. (2015) Zimbabwe District Population Projections. Government of Zimbabwe, Harare.

[8] The Sunday Mail (2019) NAC intensifies the response to HIV in Zvimba District. https://www.sundaymail.co.zw/nac-intensifies-the-response-to-hiv-in-zvimba-district

[9] Dube, S. (2017) An Evaluation of the Adverse Events following Voluntary Medical Male Surveillance System in Chegutu District, Zimbabwe. (Unpublished)

[10] Matambo, R., Duri, C., Ruhanya, V., Mungofa, S., Chadambuka, E., Nyandoro, G., et al. (2016) Evaluation of a Tuberculosis Surveillance System in the Harare City Health Department in Zimbabwe. International Journal of Tropical Disease \& Health, $14,1-10$. 
[11] Marongwe, P., Gonouya, P., Madoda, T., Murenje, V., Tshimanga, M., Balachandra, S., et al. (2019) Trust but Verify: Is There a Role for Active Surveillance in Monitoring Adverse Events in Zimbabwe's Large-Scale Male Circumcision Program? PLoS ONE, 14, e0218137. https://doi.org/10.1371/journal.pone.0218137

[12] Gadzayi, M.R., Mukuzunga, M., Chadambuka, A., Chiwanda, S., Govha, E., Gombe, N.T., et al. (2019) An Evaluation of the Adverse Events Following Immunization Surveillance System: A Case of the Oral Cholera Vaccine Mass Campaign, Chimanimani and Chipinge Districts, Zimbabwe, 2019.

https://doi.org/10.21203/rs.3.rs-64895/v1

https://www.researchsquare.com/article/rs-64895/v1 


\section{Appendix 1: Interview Questionnaire for VMMC Health Workers}

Questionnaire Number Date of interview

1) Name of health facility

\section{Personal Information}

2) Designation:

3) What is your age in completed years?

4) Observed sex of participant: Male [ ] Female [ ]

5) What is the number of years you have completed in service?

6) What is the number of years you have been involved in VMMC?

\section{System attributes}

Stability of the Surveillance System (Stability to be assessed by physically checking for availability of reporting tools e.g. AE reporting forms, VMMC forms. transportation vehiclesm, staff training plans)

7a) Have you ever been trained in VMMC and AEs following VMMC Surveillance System?

Yes [ ] No [ ]

7b) If answered "Yes" to $\mathrm{Q}_{1}$ above, specify the last time when you were trained-;

Last 12 months

Last 24 months

Last 36 months

Over 36 months

7c) If answered "No" to $\mathrm{Q}_{1}$ above, give reasons why you have never been trained in VMMC

8) If you were trained, what kind of training did you receive?

Formal

On the job

Other

9) Do you believe that the training was adequate? Yes [ ] No [ ]

10) Do you feel you still need further training? Yes [ ] No [ ]

11) Do you have adequate resources to implement the VMMC Adverse Events Surveillance System? Yes [ ] No [ ]

12) Do you have access to the following communication tools? (Availability to be physically checked)-:
i) A functional phone Yes [ ] No [ ]
ii) AE Reporting forms Yes [ ] No [ ]
iii) AE severity charts Yes [ ] No [ ]
iv) Case definition charts Yes [ ] No [ ]
v) VMMC registers Yes [ ] No [ ]
vi) AE National Guide Yes [ ] No [ ]
vii) A functional transport vehicle Yes [ ] No [ ] 


\section{Knowledge of the AEs following VMMC Surveillance System}

13) Have you ever heard about the AEs following VMMC Surveillance System? Yes [ ] No [ ]

14a) What are the objectives of the VMMC AEs Surveillance System? (Tick when appropriate answer is given)

i) To ensure the safety and quality of VMMC service provision

ii) To ensure that service providers can screen for, diagnose and document $\mathrm{A} /$ Es during and after VMMC surgery.

iii) To ensure provision of timely referrals when $\mathrm{A} / \mathrm{E}$ occur.

14b) other objective

14c) I don't know

15) When should a severe AE following VMMC be reported?

Immediately

After 24 hours

After 48 hours

Other

I don't know

16a) Can you state 5 examples of AEs following VMMC?

16b) Can you describe the criteria of grading of A/Es into Mild, Moderate and Severe?:

Mild

Moderate

Severe

17a) What do you understand about the use of coding in the classification of AEs?

17b) Can you describe the classification of coding from A to C?

i) Code $\mathrm{A}$

ii) Code $B$

iii) Code $\mathrm{C}$

\section{Simplicity of the surveillance system}

18a) Have you ever identified an Adverse Event? Yes [ ] No [ ]

18b) Did you report the case? Yes [ ] No [ ]

18c) If answered "yes" to question (18b) above, please describe the reporting system.

18d) If answered "no" to (18b) above, why did you not report the case?

19a) Have you ever completed the AE reporting forms? Yes [ ] No [ ] 
19b) If answered "yes" to question (2), how long does it normally take you to complete the VMMC Adverse reporting form? (To be assessed objectively by actually filling out the form by the researcher and noting average time it takes to complete the form)

Less than 5 minutes

5 - 10 minutes

10 - 20 minutes

More than 20 minutes

20) How do you regard the simplicity of filling in data for VMMC Adverse Events?

Very simple

Simple

Difficult

Very difficult

21) Do you think further training is required to fill in the VMMC Adverse events data? Yes [ ] No [ ]

Flexibility of the surveillance system

22) Do you think VMMC personnel want any other AEs to be monitored in the surveillance system?

Yes [ ] No []

23) Which other AEs would you like to be included in the surveillance system?

24) Specify other A/Es that are being currently monitored at this site? Yes [ ] No [ ]

25) Are there any adjustments which have been done to accommodate other AEs? Yes [ ] No [ ]

Acceptability of the surveillance system (to be assessed objectively by checking for indicators of acceptability such as completeness, quality of data, timeliness and availability of feedback reports and minutes $s$ )

26a) Do you think you are responsible for compiling data for VMMC adverse events? Yes [ ] No [ ]

26b) If answered "no" to Qn 1 above, who do you think is responsible for compiling the data?

27) Are you willing to participate in the VMMC AE surveillance system?

Not willing

Willing

Very willing

28) Are you willing to compile reports on VMMC adverse events?

Not willing

Willing 
Very willing

\section{Representativeness}

29a) Do you hard to reach areas around this health facility where eligible clients may have difficulty accessing VMMC services? Yes [ ] No [

29b) Are there any private health facilities conducting VMMC and actively reporting A/Es following VMMC?

Yes [ ] No [ ]

30) If answered "yes" to Qn 1, do you carry out mobile outreaches to these areas to ensure that clients also access VMMC including monitoring of Adverse Events? Yes [ ] No [ ]

31) If you answered "yes" to the question above, do you have AE surveillance data being reported from site in these hard to reach areas? Yes [ ] No [ ]

32) If you answered "no" to question 2 above, can you give reasons why you do not conduct the outreaches

\section{Usefulness of the surveillance system}

33) Do you think that the VMMC AEs surveillance system is useful in Zvimba District?

Yes [ ] No [ ]

34) If Yes, Give reasons

35) If No, Give reasons

36a) Do you review AE data at this facility? (To be verified by checking minutes)

Yes [ ] No [ ]

36b) If yes, how often do you have such meetings?

36c) I f no, give the reasons why you do not have review meetings?

36d) Tick applicable response:

\begin{tabular}{|c|c|c|c|c|}
\hline Has the system led to; & $\begin{array}{c}\text { Yes, to a } \\
\text { great degree }\end{array}$ & $\begin{array}{c}\text { Yes, to a } \\
\text { certain degree }\end{array}$ & $\begin{array}{l}\text { Don't } \\
\text { know }\end{array}$ & $\begin{array}{c}\text { No } \\
\text { change }\end{array}$ \\
\hline $\begin{array}{l}\text { Increased attention to AEs } \\
\text { among VMMC Personnel }\end{array}$ & & & & \\
\hline $\begin{array}{l}\text { Increased attention to } \mathrm{A} / \mathrm{Es} \\
\text { in administration }\end{array}$ & & & & \\
\hline $\begin{array}{c}\text { Initiation of specific measures } \\
\text { against } \mathrm{A} / \mathrm{Es}\end{array}$ & & & & \\
\hline
\end{tabular}

Challenges in running the VMMC AEs Surveillance System. 
37) What do you think are the reasons for possible under-reporting in this surveillance system? (Tick all that apply)

i) Unavailability of reporting forms [ ]

ii) Clients not attending reviews [ ]

iii) A complex surveillance system [ ]

iv) Lack of knowledge of AEs among HCWs [ ]

v) Weaknesses in the reporting channel [ ]

Other reasons (specify)

38) Can you list the challenges you encounter in running the surveillance system

Thank you for participating in the study

\section{List of Abbreviations}

AE: Adverse Events

AIDS: Acquired Immunodeficiency Syndrome

CDC: Center for Disease Control

DHE: District Health Executive

HSO: Health Studies Office

PMD: Provincial Medical Director

VMMC: Voluntary Medical Male Circumcision 